\title{
A Logística na Aquisição de Medicamentos pelo SUS no Município de Petrolina
}

\author{
Agnaldo da Silva Monteiro ${ }^{1}$; Cristiane Stenia Oliveira Coelho ${ }^{2}$; Danielle do Nascimento Lins ${ }^{3}$; \\ Eslany Costa de Oliveira ${ }^{4}$ :Lucimara Araújo Campos Alexandre ${ }^{5}$
}

Resumo: O presente trabalho faz um estudo sobre a logística de aquisição de medicamentos pelo SUS, no município de Petrolina, a fim de compreender como se dar o processo de aquisição desses medicamentos e a dispensação para a população em quantidade e qualidade adequada, para que os mesmos não venham a faltar e, apontar possíveis alternativas para melhoria do processo de logística na aquisição e distribuição dos mesmos.

Palavras - chave: Logística. Aquisição. Medicamentos.

\section{Logistics in Drug Purchase by SUS in the city of Petrolina}

\begin{abstract}
The present study makes a study on the logistics of the acquisition of medicines by SUS, in the city of Petrolina, in order to understand how to give the process of acquisition of these drugs and the dispensing to the population in quantity and adequate quality, so that the same Not to lack and to point out possible alternatives to improve the logistics process in the acquisition and distribution of the same.
\end{abstract}

Key words: Logistics. Acquisition. Medicines.

\section{Introdução}

A Constituição Federal de 1988, no artigo 37, cita expressamente alguns princípios que toda administração pública deve seguir, dentre eles o da eficiência, inserido pela emenda 19/98, o qual visa ampliar as oportunidades para o gestor público oferecer serviços de qualidade para a população de modo eficaz, eficiente e efetivo, ou seja, deve haver uma logística que possibilite a gestão, oferecer à população serviços de saúde com qualidade, segurança e no tempo certo (BRASIL, 1988).

\footnotetext{
${ }^{1}$ Bacharéis em Administração Pública pela Universidade Federal do Vale São Francisco - UNIVASF - Campus Juazeiro. Contatos: agnaldodasilvamonteiro@gmail.com; cris.stenia@gmail.com; lins.dani@hotmail.com; eslanycosta@hotmail.com

${ }^{2}$ Professora Orientadora da Universidade Federal do Vale São Francisco - UNIVASF - Campus Juazeiro. Contato: Lucimara.univasf@gmail.com.
} 
O medicamento é um dos componentes do tratamento de saúde que precisa ser garantido e disponibilizado a população. O Ministério da Saúde regula através de portarias a produção farmacêutica, a seleção de medicamentos que devem ser empregados pelo Sistema Único de Saúde (SUS), a aquisição, a dispensação, a política e assistência farmacêutica e o uso racional de medicamentos. "A aquisição de medicamentos é uma das principais atividades da Gestão da Assistência Farmacêutica e deve estar estreitamente vinculada às ofertas de serviços e à cobertura assistencial dos programas de saúde". Uma boa aquisição de medicamentos deve considerar primeiro o que comprar (seleção); quando e quanto comprar (programação); e como comprar. "O monitoramento e a avaliação dos processos são fundamentais para aprimorar a gestão e intervir nos problemas" (MINISTÉRIO DA SAÚDE, 2006).

O Sistema Único de Saúde (SUS) foi criado para garantir acesso universal e igualitário a todo cidadão, visando assim, a diminuição de risco de doenças e de outros agravos. De acordo com o artigo $6^{\circ}$ da Lei 8.080 de 1990, onde determina o campo de atuação do SUS está incluso, dentre outros, a assistência farmacêutica, que dá ao cidadão direito de acesso a medicamentos para as mais diversas indicações de tratamento (BRASIL, 1990).

Para adquirir os medicamentos, O Ministério da Saúde (MS) por intermédio da Secretaria Executiva/Departamento de Logística em Saúde realiza as compras de medicamentos prescritos no SUS por licitação, dispensa de licitação e inexigibilidade, caso não haja competição neste último caso. As regras desse procedimento formal estão estabelecidas principalmente nas Leis $\mathrm{n}^{\mathrm{o}} 8.666 / 1993$ e $\mathrm{n}^{\mathrm{o}} 10.520 / 2002$ para o pregão eletrônico. Em uma licitação busca-se a proposta mais vantajosa para a Administração Pública em consonância com os princípios de igualdade entre licitantes, publicidade e observância das condições exigidas no instrumento de abertura (PORTAL DA SAÚDE, 2017).

Antes de adquirir os medicamentos, para evitar desperdícios, o município deve fazer o mapeamento epidemiológico das doenças mais frequentes da cidade, levando em conta os medicamentos relacionados no RENAME (Relação Nacional de Medicamentos Essenciais) seguindo as políticas estaduais para a aquisição pelo processo licitatório, dispensa ou inexigibilidade. (RENAME, 2014) 
Nos serviços de saúde, a logística de aquisição e distribuição das medicações se apresenta como um grande desafio a ser enfrentado, pois o setor responsável deve planejar e gerir as atividades de compras, armazenagem e gerenciamento de materiais em estoque, bem como distribuir esses materiais destinados ao uso em atividades hospitalares, nas unidades de saúde, uma vez que a ausência de acompanhamento e controle de todo o processo impede a apuração das reais necessidades de abastecimento e pode ocasionar a falta ou o desperdício.

Percebe-se no município de Petrolina ingerência em relação à aquisição e distribuição de medicamentos nas unidades de saúde espalhadas por vários bairros da cidade, visto que faltam remédios de doenças consideradas de maior incidência na população pelo Ministério da Saúde, como hipertensão e diabetes, por exemplo, além de faltar frequentemente vacinas, que atuam na prevenção de doenças, principalmente nas unidades de saúde mais distantes da Secretaria Municipal de Saúde.

Considerando o exposto acima, este projeto de pesquisa tem como principal objetivo estudar a logística na aquisição e distribuição de medicamentos pelo SUS no município de Petrolina/PE, a fim de compreender como se dá o processo de aquisição desses medicamentos e a dispensação para a população em quantidade e qualidade adequada, para que os mesmos não venham a faltar, uma vez que a interrupção no tratamento além de afetar a qualidade de vida dos usuários, pode levá-los ao óbito.

Por fim, o presente estudo será de extrema importância, uma vez que poderá proporcionar ao acadêmico, experiências novas, além do aprendizado na troca de informações com a instituição onde se dará a pesquisa, visando o crescimento de ambas as partes, assim como para a gestão municipal, que a partir desse estudo poderá melhorar seu planejamento operacional no que diz respeito a assistência farmacêutica do município, visando reduzir a incidência dos principais problemas detectados nesta pesquisa, prestando a população uma assistência a saúde de qualidade. 


\section{Metodologia}

Trata-se de um estudo de abordagem qualitativa, observacional, do tipo descritivo, cuja relação entre o pesquisador e o objeto de estudo é dinâmica, particular e temporal que exige uma interpretação à luz do contexto, do tempo e dos fatos de modo imparcial (MICHEL, 2009, p.36-37).

O estudo será realizado na Farmácia da Família da Secretaria Municipal de Saúde (órgão responsável pelo gerenciamento de recursos e políticas públicas na área da saúde do município).

A coleta de dados se dará por meio de entrevista, cujos entrevistados serão, os servidores públicos responsáveis pela aquisição e distribuição de medicamentos na cidade de Petrolina/PE. Como instrumento de coleta será utilizado um questionário semiestruturado, com perguntas abertas, as quais abordarão as questões relacionadas a aquisição, armazenamento e distribuição de medicamentos.

O instrumento constou das seguintes questões: 1. Quem são os profissionais responsáveis por realizar a Logística de Medicamentos do município?; 2. Como é feito o planejamento para a compra?; 3. Como é feita a aquisição, armazenagem e distribuição desses medicamentos?; 4. Quais medicamentos o município recebe diretamente da União, e como é feito a dispensação desses?; 5. Como se dá o controle de estoque dos medicamentos?; 6. Diante do alto valor dos produtos e do fracionamento da entrega, como a Secretaria de Saúde trabalha para não deixar faltar medicamentos para a população?; 7. Por que faltam medicamentos e vacinas nas unidades de saúde?; 8. De que forma são distribuídos esses medicamentos em Hospitais e AMES do Município, e qual critério utilizado para que não haja desperdício?; 9. Existe um estoque mínimo de segurança?; 10. O que poderia melhorar na logística de aquisição, armazenagem e dispensação dos medicamentos as unidades de saúde?; 11. Por que os medicamentos para tratamento de doenças graves como Câncer, chegam a faltar nos hospitais?; 12. Em relação aos medicamentos autorizados pela justiça para disponibilização ao paciente com prazo para entrega em até 24 horas. Como é feita essa aquisição? Quais empresas fornecem esse medicamento, e de que maneira chegam até o ponto de distribuição?; 13. Quais os principais problemas, faltas e/ou fragilidades nesse processo de compra e distribuição de medicamentos no município de Petrolina? 
Após a coleta dos dados, será feito uma análise qualitativa das questões abordadas na entrevista que poderão levar a um esclarecimento mais aprofundado de como se dá à aquisição e distribuição de medicamentos em toda a Rede Hospitalar e AMES (Ambulatório Médico de Especialidades) do município, analisando desde o planejamento do quantitativo que se deseja para atender a demanda da população até a distribuição nas unidades de saúde deste município, levando em conta o orçamento da secretaria de saúde, as medicações que o município recebe dos governos estadual e federal, que não passam pelo processo licitatório nem demandam gastos extras até a dispensação desses medicamentos.

Os resultados dos dados pesquisados serão apresentados em relatório de forma clara e concisa, de acordo com o material coletado e estudado, prezando pela imparcialidade, transparência e idoneidade das análises.

Os objetivos do presente estudo foram: descrever a logística de aquisição e distribuição de medicamento pelo SUS, no município de Petrolina-PE; descrever o processo de aquisição, armazenamento e dispensação de medicamentos; identificar e registrar os principais problemas na logística de aquisição e distribuição dos medicamentos; e, apontar possíveis alternativas para melhoria do processo de logística na aquisição e distribuição dos medicamentos.

\section{Resultados e Discussão}

A entrevista foi realizada com o farmacêutico responsável pela Farmácia da Família que coordena o processo de aquisição de medicamentos por meio da Central de Abastecimento Farmacêutico-CAF juntamente com a diretoria e outros farmacêuticos do município. O planejamento é realizado a partir de um sistema gratuito chamado HORUS do Ministério da Saúde, fornecido pelo DATASUS- Sistema de Informática do SUS que informa todo processo logístico desde a aquisição até a distribuição dos medicamentos.

A aquisição é feita através de processo licitatório, que é antecedido pela seleção e padronização dos medicamentos, concluída a compra, após a entrega pelos fornecedores esses 
medicamentos são armazenados na CAF e a partir daí são distribuídas para as unidades de saúde do município de Petrolina.

Segundo o coordenador nenhum medicamento é entregue diretamente pelo Governo Federal, mas há uma contrapartida por meio de recursos financeiros de $\mathrm{R} \$ 5,10$ por habitante. Tanto os Estados como os municípios utilizam a RENAME para relacionar o nome dos medicamentos que serão necessários para determinado período. Esse processo eles chamam de padronização, pois só podem comprar remédios que estão nessa lista. O planejamento para aquisição dos medicamentos em Petrolina é baseado no consumo médio mensal dos medicamentos e o suprimento durante o tempo necessário até a chegada novamente dessas medicações que são chamadas de estoque de reposição- que é equivalente ao estoque de segurança.

A aquisição de medicamentos é realizada separadamente das vacinas, estas através do Programa Nacional de Imunização- PNI e dentro do munícipio Programa Municipal de Imunização-PMI, a Assistência Farmacêutica é responsável apenas pela aquisição dos medicamentos. A principal dificuldade de suprimento dessas medicações no município é devido ao baixo valor repassado pela União ( $\mathrm{R} \$ 5,10)$ que não é suficiente para a demanda do município e este deve conseguir recursos para complementar, mas nem sempre é possível e ocasiona a falta desses remédios a população, pode ocorrer também devido a falha humana na gestão desse planejamento, visto que são milhares de remédios que compõem a lista do RENAME.

A atenção Básica de Saúde é atendida pelo município e os hospitais, classificados de média e alta complexidade, são de responsabilidades dos Estados e da União em parceria com o município e os tercerizados. Em Petrolina, por exemplo, a gestão do Hospital Dom Malam é realizada pelo IMIP e a gestão do Hospital de Urgências e Traumas é da Universidade do Vale do São Francisco-Univasf, fundação pertencente ao Governo Federal. A Secretaria de Saúde do Município tem um programa chamado de NASF-Núcleo de Assistência à Saúde da Família que participa do processo de distribuição de medicações.

Os farmacêuticos fazem os pedidos dos medicamentos das unidades de saúde do município a CAF, esta realiza a separação de acordo com o cronograma mensal e depois distribui às unidades que ficam sob a responsabilidade de farmacêutico a gestão e o controle da distribuição a população dos bairos ondem situam-se as AMEs, Unidades de saúde e a 
farmácia da Família. Para a compra de medicamentos para o tratamento do câncer, assim como do suprimento hospitalar na questão dos medicamentos há uma verba específica em que cada ente estadual ou federal ficará responsável pela execução, cabendo ao município a cobrança da aplicação do recurso em medicamentos para o tratamento do câncer para os pacientes que necessitam desse atendimento.

A assistência farmacêutica se subdivide em estratégica e especializadaresponsabilidade do Estado/União e básica que pertence ao município. Muitas vezes falta informação da população e a alguns funcionários públicos que trabalham na ponta e não conhece todo trâmite de distribuição das medicações especializadas que tem um alto custo para o Estado, não pelo valor do remédio, mas sim pelo uso prolongado desse tratamento. As pessoas, muitas vezes, procuram o judiciário para ter seu direito garantido ao remédio, porém nem sempre buscam informações no local correto sobre a obtenção da sua necessidade. Em Pernambuco, há a farmácia do Estado que distribue remédios especializados, mas há medicações que o governo não é obrigado a fornecer por não está no RENAME e nesse aspecto há a judicialização, ou seja, a pessoa busca à justiça para garantir que o Estado forneça a medicação específica solicitada pelo médico para tratamento do paciente.

Os gestores municipais estão trabalhando para que não faltem medicamentos a população, incluindo ações de informações aos cidadãos para inclusão de remédios na lista do RENAME através de consultas públicas, porém ainda está pouco popularizado, principalmente para a população usuária e dependente de medicamentos fornecidos pelo Poder Público.

Percebe-se no site da Prefeitura Municipal de Petrolina que nem todos os dados da gestão da cidade estão disponibilizados, dificultando assim o controle social e informações claras para maior entendimento da população. A lei 12527/2011 e o decreto 7.724/2012 regulamentam o acesso à informação, porém para por em prática, necessita de um sistema de informação para tratar os dados e disponibilizar as informações sobre o funcionamento da máquina pública, o que pouco ocorre nos municípios de menor porte do Brasil. (TCU, 2011)

A atenção básica disponibilizada por meio de políticas públicas na área da saúde pode determinar a qualidade dessa logística no município em questão. O empenho em melhorar a cada dia é recurso insubstituível, pois é preciso ainda ser feito muito mais, para se poder alcançar uma boa qualidade dos serviços prestados a sociedade. Ainda que as verbas sejam 
limitadas, e a falha humana aconteça, o responsável por garantir a disponibilização desses medicamentos, é o município, seja executando, seja cobrando do Estado.

\section{Conclusões}

A saúde é um dos pilares a qualidade de vida de uma sociedade, e sabendo disso, o governo por meio de políticas sociais tem buscado ampliar e melhorar a Logística de medicamentos. Pela Constituição Federal, todo município deve prover para sua população uma excelência na gestão, na busca da supremacia do interesse público. Buscar a melhor proposta não é somente prerrogativa da administração pública seja ela municipal ou estadual, mas uma escolha na concretização de ações que possam garantir um processo menos demorado para aquisição, armazenagem e distribuição desses medicamentos.

Infelizmente, a restrição orçamentária e financeira no setor público limita a prestação de serviços de melhor qualidade aos cidadãos, principalmente para aqueles que dependem de ação direta dos governantes. É necessário um sistema de informação eficiente que monitore todo processo logístico desde a compra até a entrega de medicamentos aos cidadãos, de modo que a sociedade fique informada e possa participar desse processo dando um feedback por meio de avaliações para que haja um processo de melhoramento contínuo.

É importante maior flexibilidade na gestão dos próprios recursos, reduzindo a prática de contingenciamento dos mesmos de forma que possam estar disponíveis de acordo com a programação anual para a existência de um planejamento eficaz. E também uma mudança cultural dos servidores públicos, para que os incentivos e punições estejam atrelados aos resultados individuais e coletivos alcançados.

Existem várias ferramentas de suporte e uma delas é o RENAME, aonde é possível identificar quais medicamentos são necessários para atender a saúde básica, ela permite ao profissional de farmácia, fazer uma busca na escolha de todos os itens importantes e indispensáveis para atender as necessidades e as demandas do município. Sabe-se que todos esses procedimentos vão exigir bom senso, coerência, conhecimento da área de atuação, para assim desenvolver todo processo de compra desses medicamentos, sem que haja ausência ou falta deles, evitando assim todo e qualquer desperdício. 
Sendo assim, a cooperação entre as esferas do governo deve ser mútua, cada qual com sua autonomia, com um objetivo único de agregar valor à vida de cada cidadão que busca um tratamento digno de saúde para si, ou para alguém da sua família.

\section{Referências}

BRASIL, Constituição (1988). Constituição da República Federativa do Brasil, Brasília, DF: Senado Federal: Centro Gráfico, 1988. P. 20-22.

BRASIL, Controladoria-Geral da União. (2011). Acesso à Informação Pública: Uma introdução a Lei 12.527, de 18 de novembro de 2011. Brasilia.

BRASIL. Lei n o 8.080, de 19 de setembro de 1990 - Planalto. Disponível em: www.planalto.gov.br/ccivil. Acessado em: 12/11/2016, às 17:50.

MERÍSIO A.; Kleba M. E.; Silva, N. C. da; Kovalesk, D. F. A aquisição de medicamentos para o Sistema Único de Saúde em municípios de pequeno porte do Estado de Santa Catarina, Santa Catarina, Rev. Bras. Farm. 93(2): 173-178, 2012. Disponível em: http://www.rbfarma.org.br/files/rbf-201293-2-7.pdf, acessado em 30/01/2016.

MICHEL, Maria Helena. Metodologia e Pesquisa Científica em Ciências Sociais. 2. Ed. atual.e amp. São Paulo: Atlas, 2009.

MINISTÉRIO DA SAÚDE, Aquisição de Medicamento para Assistência Farmacêutica no SUS,Brasília. DF, 2006.

MINISTÉRIO DA SAÚDE. RELAÇÃO NACIONAL DE MEDICAMENTOS ESSENCIAIS : RENAME 2014 /, Secretaria de Ciência, Tecnologia e Insumos Estratégicos, Departamento de Assistência Farmacêutica e Insumos Estratégicos. - 9. ed. rev. e atual. - Brasília : Ministério da Saúde, 2015.

PORTAL DA SAÚDE. Profissional e Gestor. Disponível em: $<$ http://portalsaude.saude.gov.br/index.php?option=com content\&view=article\&id=6749>. Acesso em 10 de junho de 2017.

RAP - Revista de Administração Pública. Rio de Janeiro45(1): 107-39 jan./fev. 2011 ISSN 0034-7612. Disponível em: http://www.scielo.br/pdf/rap/v45n1/v45n1a06.pdf. Acesso em: 06 set. 2016.

ROSA, Rodrigo de Alvarenga. Gestão de operações e logística I / Rodrigo de Alvarenga Rosa. Florianópolis: Departamento de Ciências da Administração / UFSC; [Brasília]: CAPES: UAB, 2011.160p.

Como citar este artigo (Formato ABNT):

MONTEIRO, Aguinaldo da S.; COELHO, Cristiane S.O.; LINS, Danielle do N.; OLIVEIRA, Eslany C de; ALEXANDRE, Lucimara A.C. A Logística na Aquisição de Medicamentos pelo SUS no Município de Petrolina. Id on Line Revista Multidisciplinar e de Psicologia, 2017, vol.11, n.37, p.37-45. ISSN: 1981-1179.

Recebido: 07.08.2017;

Aceito: 09.08.2017 
Id on Line Revista Multidisciplinar e de Psicoloqia

Id on Line Multidisciplinary and Psycology Journal 